\title{
The center conjecture for equifacetal simplices
}

\author{
Allan L. Edmonds \\ (Communicated by R. Scharlau)
}

\begin{abstract}
An equifacetal simplex, in which all facets are congruent, has a unique center. The center conjecture states that a simplex that has a unique center must be equifacetal. A strong version of the conjecture is proved in dimensions at most six by showing that there is an explicit list of centers, defined for all simplices, whose coinciding implies the simplex is equifacetal. It remains an open problem whether the conjecture is true in dimensions greater than six.
\end{abstract}

\section{Introduction}

A geometric simplex in euclidean space is said to be equifacetal if all its facets are congruent to one another. In an earlier paper [3] the author developed an approach to the existence and classification of equifacetal $d$-simplices in terms of what we here call the colored-graph type, consisting of an edge-coloring of the complete graph $K_{d+1}$, colored by the lengths of the corresponding edges of the simplex, and the partition type, consisting of the partition of $d$ given by the numbers of edges of each length incident at a given vertex.

An important point in the previous paper is the observation that the isometry group of an equifacetal simplex acts transitively on the vertices. This shows that the partition type is well-defined. In fact the vertex-transitivity condition completely characterizes equifacetal simplices.

In the earlier paper we showed that an equifacetal simplex has a unique center, just like an equilateral simplex, in which all edges have the same length. In dimension 2 it is a well known, classical fact that if two traditional centers (centroid, circumcenter, incenter, orthocenter) of a given triangle coincide, then the triangle is equilateral. The existence of equifacetal, non-equilateral, simplices in all higher dimensions shows that uniqueness of centers cannot imply that a simplex is equilateral. But it then becomes natural to conjecture that a simplex that has a unique center must indeed be equifacetal.

The full meaning of the conjecture depends upon giving a clear definition of simplex center, which we do in Section 3. 
It will be shown that there is a sequence of simplex centers

$$
P^{0}(S), P^{1}(S), \ldots, P^{d}(S), \ldots
$$

which we call perimeter points, defined and continuous for all simplices, such that if $d \leq 6$ then a $d$-simplex $S$ for which the centers $P^{0}(S), P^{1}(S), \ldots, P^{d}(S)$ coincide with the incenter is equifacetal. In all dimensions $d$ the condition that $P^{0}(S)=P^{1}(S)=$ $\cdots=P^{d}(S)$ (without reference to the incenter) is equivalent to a condition in general weaker than equifacetality, which we call vertex-uniformity, where every vertex has the same multiset of edge lengths of edges emanating from it. Full definitions are given in subsequent sections. It remains a conjecture that the restriction to dimensions $d \leq 6$ above may be removed.

Work on related matters, especially concerning simplices all of whose facets have the same area, may be found in V. Devidé [2], P. McMullen [8], H. Martini and W. Wenzel [7], and B. Weissbach [10]. In related work the recent paper of S. Krantz, J. McCarthy, and H. Parks [6] explores the question of when alternative notions of the centroid coincide.

\section{Vertex transitivity and vertex uniformity}

In this section we summarize basic definitions and results from [3].

2.1 Background definitions. A $d$-simplex $S$ is the convex hull of $d+1$ affinely independent points in some Euclidean space $\mathbb{R}^{n}, n \geq d$. Given a labeling of its vertices $A_{1}, \ldots, A_{d+1}$ we may view $S$ as an element of $\left(\mathbb{R}^{n}\right)^{d+1}$. We also denote the convex hull simply by $\left[A_{1}, \ldots, A_{d+1}\right]$. Often we do not distinguish between a simplex and a list of its vertices. For convenience we often assume without loss of generality that a given $d$ simplex lies in $\mathbb{R}^{d}$. But such an assumption is too restrictive in general, since we need to consider the facets of a simplex as simplices as well.

A simplex is equifacetal if all its facets (i.e., codimension one faces) are congruent.

An edge-coloring of a graph is an assignment of labels to the edges, with no further requirement such as edges incident at a vertex having different colors. An edge-colored graph is equifacetal if all its facets (i.e. result of deleting one vertex and all edges incident at it) are colored graph isomorphic. A colored graph has a colored graph automorphism group consisting of graph isomorphisms that preserve the coloring.

A simplex is vertex-uniform if the multisets of lengths of edges incident at a vertex are the same for all vertices. Similarly an edge-colored graph is vertex-uniform if the multisets of colors of edges incident at a vertex are the same for all vertices.

A simplex is vertex-transitive if its isometry group acts transitively on the set of vertices. Similarly, an edge-colored graph is vertex-transitive if its colored graph automorphism group acts transitively on the set of vertices.

2.2 Background results. A $d$-simplex gives rise to an edge-coloring of its 1-skeleton, the complete graph $K_{d+1}$ on $d+1$ vertices, whose colored graph automorphism group is isomorphic to the isometry group of the simplex. An equifacetal $d$-simplex gives rise to an equifacetal edge-coloring of the complete graph $K_{d+1}$. 
Any edge-coloring of $K_{d+1}$ arises from a suitable $d$-simplex (with lengths corresponding to the various colors chosen close to 1 , say).

An equifacetal simplex or edge-colored complete graph is known to be vertex-transitive. See Edmonds [3]. The converse is trivial. Because of this, we will sometimes refer to equifacetal simplices or graphs as vertex-transitive.

Because an equifacetal simplex or edge-colored complete graph is vertex-transitive, it is necessarily also vertex-uniform. But vertex-uniformity does not in general imply a simplex is equifacetal, in dimensions greater than four.

A vertex-uniform edge-colored graph in which each vertex has total valence $d$ induces a well-defined partition of $d$ given by the valences of the various graphs of constant color.

When $d$ is odd every partition of $d$ is realized by a vertex-uniform edge-coloring of the complete graph $K_{d+1}$. But not every partition arises from a vertex-transitive edgecoloring of $K_{d+1}$ or an equifacetal simplex. When $d$ is even, the only additional condition required for a vertex-uniform realization is that the terms of the partition should be even. See [3]. Every such even partition is realized by an equifacetal simplex, but there are also vertex-uniform realizations that are not equifacetal. (When $d$ is odd there are deeper restrictions on the partitions that arise from equifacetal simplices that are analyzed definitively in a recent paper of the author [4].)

\section{Formulation of the center conjecture}

We need a precise and unambiguous definition of the term "simplex center".

3.1 Simplex centers. Consider $d$-simplices lying in $\mathbb{R}^{n}$. A center function is a map $\left(\mathbb{R}^{n}\right)^{d+1} \rightarrow\left(\mathbb{R}^{n}\right)$ satisfying certain properties, including equivariance with respect to isometries of $\mathbb{R}^{n}$ and invariance under permutation of vertices. A center of a simplex is then the result of evaluating a center function on the simplex. A bit more formally we have the following.

Definition 1. By a $d$-simplex center function, we understand a continuous function

$$
\varphi: \mathbb{R}^{n} \times \mathbb{R}^{n} \times \cdots \times \mathbb{R}^{n} \rightarrow \mathbb{R}^{n}
$$

$(d+1$ factors in the domain, not necessarily defined on the entire domain, but defined at least on the space of affinely independent $(d+1)$-tuples) such that for any $d$-simplex $\left[A_{1}, A_{2}, \ldots, A_{d+1}\right]$ the following properties hold:

(1) Span. $\varphi\left(A_{1}, A_{2}, \ldots, A_{d+1}\right)$ is contained in the affine span of $A_{1}, A_{2}, \ldots, A_{d+1}$.

(2) Permutation Invariance.

$$
\varphi\left(A_{1}, A_{2}, \ldots, A_{d+1}\right)=\varphi\left(A_{\sigma(1)}, A_{\sigma(1)}, \ldots, A_{\sigma(d+1)}\right)
$$

for any permutation $\sigma$ of $(1,2, \ldots, d+1)$.

(3) Isometry Equivariance.

$$
\varphi\left(T A_{1}, T A_{2}, \ldots, T A_{d+1}\right)=T \varphi\left(A_{1}, A_{2}, \ldots, A_{d+1}\right)
$$

for any isometry $T: \mathbb{R}^{n} \rightarrow \mathbb{R}^{n}$. 
One might reasonably also impose a condition about the behavior of a center function with respect to similarity. Similarly, one could allow center functions that are not defined for all simplices, such as the orthocenter in higher dimensions. But such a condition plays no role in the present work.

Up to isometry any $d$-simplex may be assumed to lie in the standard $\mathbb{R}^{d} \subset \mathbb{R}^{n}$. Thus for the purpose of defining a center one could assume that $n=d$. But on occasion one wishes to consider the centers of facets of a simplex, so it is better to have the slightly more general framework where $d \leq n$.

Definition 2. A point $X$ in the affine span of a $d$-simplex $S$ is a center of $S$ if there is a $d$-simplex center function $\varphi$ such that $X=\varphi(S)$.

Lemma 3.1. The set of centers of a simplex is an affine subspace of the affine span of the simplex.

Proof. It suffices to show that if $X$ and $Y$ are centers of a $d$-simplex $S$, then so is any affine linear combination $Z=t X+(1-t) Y$. Suppose $X=\varphi(S)$ and $Y=\psi(S)$ for center functions $\varphi$ and $\psi$. Define $\chi=t \varphi+(1-t) \psi$. Then it is clear that $\chi$ is a center function, and $\chi(S)=Z$.

Lemma 3.2. The set of centers of a simplex is a subset of the fixed point set of its group of isometries (viewed as an affine linear subspace of the affine span of the simplex).

Proof. This is an immediate consequence of the required equivariance of a center function with respect to isometries of $\mathbb{R}^{d}$.

3.2 The center conjecture. Here we begin by showing that the isometry group of an equifacetal simplex fixes just one point (in the affine span of the simplex).

Lemma 3.3. The fixed point set of the isometry group of a simplex is a single point if and only if the group acts transitively on the set of vertices.

Proof. In general the isometry group of a simplex fixes pointwise an affine plane of some dimension containing the centroid. First suppose the isometry group acts transitively on the vertices. It then follows that all the barycentric coordinates of a fixed point must be equal. Thus any fixed point must coincide with the centroid. On the other hand, suppose that the isometry group does not act transitively on the vertices. Then it has a certain number of orbits of vertices. Each such orbit corresponds to a face of some dimension and the isometry group then fixes the centroid of each such face. It follows that the isometry group fixes pointwise the affine span of the these centroids. In particular the fixed point set must be more than just one point.

By Lemmas 3.3 and 3.2 we have the following result.

Proposition 3.4. An equifacetal simplex has a unique center. 
We propose the following fundamental conjecture asserting the converse of the proposition.

Center Conjecture Version 1. If a simplex has a unique center, then it is equifacetal.

One equivalent way of phrasing the conjecture is that a simplex whose isometry group is not transitive on the vertices admits a center distinct from the centroid. This version of the conjecture is still a bit too vague.

3.3 The Strong Center Conjecture. A stronger version of the center conjecture would ask for centers that are independent of the particular simplex under consideration.

Center Conjecture Version 2 (Strong Center Conjecture). For each dimension $d$ there is a finite list of $d$-simplex center functions $\varphi_{1}, \varphi_{2}, \ldots$, defined for all simplices, such that a d-simplex $S$ is equifacetal if and only if $\varphi_{1}(S)=\varphi_{2}(S)=\cdots$.

In the next section we propose such a concrete list of (partially) new center functions.

\section{Perimeter centers and vertex-uniform simplices}

Let $S=\left[A_{1}, A_{2}, \ldots, A_{d+1}\right]$ be a $d$-simplex. Let $e_{i j}=\left\|A_{i}-A_{j}\right\|$ denote the length of the $(i, j)$ edge. Define the perimeter of a simplex $S$ to be $\operatorname{Perim}(S)=\sum_{i<j} e_{i j}$, the sum of all its edge lengths.

Let $F_{i}$ denote the facet opposite vertex $A_{i}$. We define the perimeter point to be the linear combination of the vertices weighted by the perimeter of the opposite facet, and suitably normalized to give barycentric coordinates.

Definition 3. The perimeter point of a $d$-simplex $S$ is

$$
P(S):=\sum_{i=1}^{d+1} \operatorname{Perim}\left(F_{i}\right) A_{i} /(d-1) \operatorname{Perim}(S) .
$$

Note that $\sum \operatorname{Perim}\left(F_{i}\right)=(d-1) \operatorname{Perim}(S)$, since each edge appears in $d-1$ facets, so the definition gives well-defined barycentric coordinates. It is easy to check that the definition satisfies the requirements for being a simplex center.

The perimeter point is well-defined by this formula, even for degenerate simplices, as long as not all the vertices coincide. In general the perimeter point lies in the convex hull of the vertices. Therefore, when all vertices coincide one can still define the perimeter point to be the unique vertex. In this way the perimeter point is defined and continuous on the space of all $d$-simplices, nondegenerate or not. The perimeter point of a nondegenerate simplex lies in its interior.

If $d=2$, then $P(S)$ is just the incenter. For an equifacetal simplex, $P(S)$ is explicitly the centroid, since all the barycentric coordinates are then equal (and of course we know that all centers coincide for equifacetal simplices).

This center has analogues involving $k$ th powers of lengths. Define the $k$-perimeter of simplex $S$ to be $\operatorname{Perim}^{k}(S)=\sum_{i<j} e_{i j}^{k}$. 
Definition 4. The $k$-perimeter point of a $d$-simplex $S$ is

$$
P^{k}(S):=\sum \operatorname{Perim}^{k}\left(F_{i}\right) A_{i} /(d-1) \operatorname{Perim}^{k}(S) .
$$

Then, for example, $P^{0}(S)=G$, the centroid. Again the $k$-perimeter points are simplex centers, defined and continuous on the space of all $d$-simplices, nondegenerate or not. All of these centers have strictly positive barycentric coordinates, in the case of a nondegenerate simplex, and hence lie in the interior of the simplex.

V. Devidé [2] proved that the centroid of a simplex $S$ coincides with its circumcenter if and only if the sum of the squares of the edge lengths of the facets is constant. In the present notation this amounts to the condition that $P^{2}(S)=P^{0}(S)$.

It is tempting to conjecture that a $d$-simplex $S$ for which $P^{0}(S)=P^{1}(S)=\cdots=$ $P^{d}(S)$ is equifacetal. This turns out to be too strong, but something remarkably close turns out to be true.

Theorem 4.1. A d-simplex $S$ is vertex-uniform if and only if $P^{0}(S)=P^{1}(S)=\cdots=$ $P^{d}(S)$.

Proof. If $S$ is vertex-uniform, then $\operatorname{Perim}^{k}\left(F_{i}\right)=\operatorname{Perim}^{k}\left(F_{j}\right)$ since each facet involves the same multiset of edge lengths. It follows that the barycentric coordinates of $P^{k}(S)$ are all equal, implying that $P^{k}(S)=G$ for $k=1, \ldots, d+1$. Here $G$ denotes the centroid of $S$. Since $G=P^{0}(S)$, it follows that $P^{0}(S)=P^{1}(S)=\cdots=P^{d}(S)$, as required.

Now consider the converse. Since $P^{k}(S)=P^{0}(S)$, it follows that

$$
\operatorname{Perim}^{k}\left(F_{1}\right)=\operatorname{Perim}^{k}\left(F_{2}\right)=\cdots=\operatorname{Perim}^{k}\left(F_{d+1}\right) .
$$

Thus the $k$ th power sums of the edge lengths of the facets are constant. This implies that the power sums of the edge lengths emanating from one vertex coincide with the power sums of the edge lengths emanating from any other vertex. Proposition 4.2 below then implies that the actual multisets of edge lengths at each vertex are the same.

Proposition 4.2. Let $x_{1}, \ldots, x_{n}$ and $y_{1}, \ldots, y_{n}$ be elements in a field $K$. Suppose that for $1 \leq p \leq n$ we have $\sum x_{i}^{p}=\sum y_{i}^{p}$. Then $\left\{x_{1}, \ldots, x_{n}\right\}=\left\{y_{1}, \ldots, y_{n}\right\}$ as sets with multiplicities.

Proof. Note that standard symmetric function theory (see van der Waerden [9], Section 5.7, for example) shows that since the power sums agree for the two sets of quantities, so do the elementary symmetric functions of the same quantities.

If some $x_{i}$ equals some $y_{j}$, then induction on $n$ proves the result. Consider the polynomial in $x_{1}$ given by $\prod\left(x_{1}-y_{j}\right)$. The coefficients of the powers of $x_{1}$ are the elementary symmetric functions of the $y_{j}$. But these are exactly the same elementary symmetric functions of the $x_{j}$. It follows that $\prod\left(x_{1}-y_{j}\right)=\prod\left(x_{1}-x_{j}\right)$. But the latter is obviously 0 . Therefore, since we are working over a field, some $y_{j}=x_{1}$, as required. 
4.1 The strong center conjecture in dimensions at most 4. We point out that the Strong Center Conjecture holds in dimensions at most four because in these dimensions vertex-uniformity implies vertex-transitivity.

Corollary 4.3. A $d$-simplex $S, d \leq 4$, for which $P^{0}(S)=P^{1}(S)=\cdots=P^{d}(S)$ is equifacetal.

Proof. In these dimensions one easily shows that each allowable partition type (even if $d$ is even) is realized by an essentially unique vertex-uniform edge-coloring of $K_{d+1}$, and the coloring is in fact vertex-transitive, and hence is given by an equifacetal simplex.

In dimensions 5 and higher, however, there are vertex-uniform $d$-simplices that are not vertex-transitive. For example, one can realize the partition $[1,1,1,1,1]$ by a vertexuniform edge-coloring of $K_{6}$. But no such coloring is vertex-transitive. See Subsection 6.2.

\section{The incenter and the equiareal equations}

If the Strong Center Conjecture is to hold in general it will be necessary to involve other centers beyond the perimeter points that determine only vertex-uniformity.

5.1 Incenter. Numerical explorations indicated that the incenter may be the appropriate additional center. Theorem 4.1 reduces the Strong Center Conjecture to the following.

Center Conjecture Version 3 (Precise version of Strong Center Conjecture). A d-simplex $S$ that is vertex-uniform and whose incenter coincides with the centroid is equifacetal.

Vertex uniformity actually implies that the circumcenter $C$ coincides with the centroid $G$, as follows from work of Devidé [2].

We may therefore assume that the vertices of $S=\left[A_{1}, \ldots, A_{d+1}\right]$ lie on the sphere of radius $R$ centered at the origin $O$. So $\left|A_{1}\right|=\cdots=\left|A_{d+1}\right|=R$ and $\sum A_{i}=O$. This takes care of $C=G=O$. Now consider the incenter $I$. The condition that $C=I$ is known to be equivalent to equiradiality, that is, the condition that all facets have the same circumradius. See Devidé [2], McMullen [8], or Edmonds-Hajja-Martini [5]. The condition that $G=I$ is known to be equivalent to equiareality, that is the condition that all facets have the same $(d-1)$-volume.

We will focus on the equiareal condition, which seems somewhat easier to deal with, and will therefore need to review how to compute the volumes of the facets in terms of the edge lengths. 
5.2 Volume and the equiareal equations. For more details of a modern treatment of the computation of the volume of a simplex in terms of the lengths of its edges, see Berger [1], for example. Suppose $S=\left[A_{1}, \ldots, A_{d+1}\right]$ is a $d$-simplex. Let $M=\left[m_{i j}\right]$ be the symmetric $(d+1) \times(d+1)$ matrix where $m_{i j}=\left\|A_{i}-A_{j}\right\|^{2}$. Now let $M_{+}$be the $(d+2) \times(d+2)$ "Menger matrix"

$$
M_{+}=\left[\begin{array}{cccc}
0 & 1 & \ldots & 1 \\
1 & & & \\
\vdots & & M & \\
1 & & &
\end{array}\right]
$$

It is a standard fact that, up to a multiplicative constant that depends only on the dimension, $\operatorname{det} M_{+}$gives the square of the $d$-volume of $S$, namely:

$$
V^{2}=\frac{(-1)^{d+1}}{2^{d}(d !)^{2}} \operatorname{det} M_{+}
$$

The $(d-1)$-volume of the $i$ th facet of $S$ is thus given by

$$
V_{i}^{2}=\frac{(-1)^{d}}{2^{d-1}((d-1) !)^{2}} \operatorname{det} M_{+}^{(i)}
$$

where $M_{+}^{(i)}$ is obtained from $M$ by replacing each entry of the $i$ th row and $i$ th column, except the diagonal entry, by 1 , and the diagonal entry by 0 . The equiareal equations then become

$$
\operatorname{det} M_{+}^{(1)}=\operatorname{det} M_{+}^{(2)}=\cdots=\operatorname{det} M_{+}^{(d+1)} .
$$

5.3 Remarks on computation. The results in the next two sections, verifying the Strong Center Conjecture in dimensions 5 and 6, depend on calculations assisted by the computer algebra system Maple. The computations could at least in principle be done by hand, rising to the level of working with equations obtained from taking determinants of numerous 6 by 6 and 7 by 7 matrices with 5 or 3 unknowns in them, combining the expressions in appropriate ways, and judiciously simplifying the resulting equations. The equations in question turn out to be reducible to degrees 1 and 2. We have tried to summarize the approach in the main text. Although it is not appropriate to include here the actual Maple code used, it is available from the author upon request.

\section{The strong center conjecture in dimension 5}

We will use a detailed case-by-case analysis to prove the precise version of the Strong Center Conjecture in dimension 5. 
6.1 Some terminology. We introduce some convenient general terminology as follows. If $\left[d_{1}, \ldots, d_{p}\right]$ is a partition of $d$ arising from a vertex-uniform edge-coloring of the complete graph $K_{d+1}$, then we refer to the collection of edges corresponding to $d_{i}$ as a $d_{i}$-part. If $d_{i}=1$, for example, then a 1-part is a disjoint collection of edges containing all vertices. In graph theory this is sometimes called a 1 -factor. If $d_{i}=2$, for example, then a 2-part is a disjoint union of cycles containing all vertices, each cycle of length at least 3 . In general we will refer to a 2-part as a chain made up of cycles. If the edge-coloring is actually vertex-transitive then all the cycles in a 2-part have the same length. If $d<5$, then the only possible 2-part is a $(d+1)$-cycle. When $d=5$, there are two possible 2-parts: a 6-cycle or a pair of disjoint 3-cycles. As the dimension grows the number of possible configurations grows rapidly.

We will say that one vertex-uniform edge-coloring is a specialization of another if the first is obtained by recoloring some distinct parts of the second so that they become colored the same. The partition associated with the second edge-coloring is obtained by combining some of the entries in the partition associated with the first edge-coloring.

The process inverse to specialization will called splitting. This is more delicate and will only be used in the case of a 2-part with the property that all of its component cycles have even length. Then one can split the 2-part into two 1-parts by recoloring every other edge along the cycles.

6.2 Vertex uniform simplices in dimension 5. Here we describe the possible vertexuniform realizations of all the partitions of 5 pointing out which are equifacetal (=vertextransitive) and which not. We proceed systematically through the partitions of $d=5$ :

I. Partition type $[1,1,1,1,1]$ : There is a unique realization by an edge-colored $K_{6}$, but it is not equifacetal. Note that any two 1-parts fit together to form a 6-cycle, unique up to relabeling. Then there is a unique way, up to cyclic permutation and reflection, to fit in a second 6-cycle corresponding to another two 1-parts. This leave an essentially unique final 1-part. The resulting edge-coloring is visibly not vertex-transitive, however.

II. Partition type $[2,1,1,1]$. There are two realizations. One is not equifacetal, and one is equifacetal. In the first case the 2-part is a single 6-cycle. If this were vertex-transitive, then so would be the result of splitting the 6-cycle into two 1-parts. But this would realize $[1,1,1,1,1]$ by an equifacetal simplex. In the second case, the 2-part is the disjoint union of two 3-cycles. Now two of the 1-parts fit together to form an essentially unique 6-cycle. There is a unique way to add a pair of 3-cycles. And what is left over gives the last 1-part. The resulting edge-coloring is visibly vertex-transitive.

III. Partition type $[2,2,1]$. There are two realizations. One is not equifacetal, and one is equifacetal. In the first case both 2-parts form 6-cycles. This one is necessarily a specialization of $[1,1,1,1,1]$. For the second case there is also a realization with one 2-part a 6-cycle and the other 2-part a pair of 3-cycles. It is equifacetal. Finally, we note that it is impossible that both 2-parts correspond to pairs of 3-cycles. To see this, observe that deleting a disjoint pair of 3-cycles leaves a 6-cycle with antipodal vertices connected by edges. There are no 3-cycles in such a graph!

The vertex-uniform realizations of all remaining partitions are necessarily essentially unique and are equifacetal: 
IV. Partition type $[3,1,1]$. The two 1-parts fit together to form a 6-cycle. All other edges are colored the same. So there is a unique vertex-uniform realization and on the other hand it is visibly vertex transitive.

V. Partition type $[3,2]$. There are two possible vertex-uniform realizations and they are both vertex-transitive hence equifacetal. The 2-part can be a single 6-cycle with everything else colored 3; or the 2-part can be a pair of 3-cycles with everything else colored 3.

VI. Partition type $[4,1]$. Three disjoint edges colored 1; everything else colored 4. Clearly vertex-transitive, hence equifacetal.

VII. Partition type [5]. This is the equilateral case.

6.3 Equiareal equations in dimension 5. Here we apply the preceding case-by-case analysis to show that a vertex-uniform and equiareal (or equiradial) simplex is equifacetal. We literally write down expressions for the areas of the facets and see what conditions on the edge lengths are implied by equiareality.

Maple calculations discussed below show that a specialization of a vertex-uniform realization of $[1,1,1,1,1]$ that is also equiareal must be a specialization of $[3,1,1]$. It will follow that the vertex-uniform non-equifacetal realizations of $[1,1,1,1,1],[2,1,1,1]$, and $[2,2,1]$ are not equiareal. The remaining realizations are all known to be equifacetal.

According to the above discussion of vertex-uniform 5-simplices, the realizations that are not equifacetal are specializations of the essentially unique realization of $[1,1,1,1,1]$. The symmetric matrix $M$ of squares of edge lengths (labelled by short hand for the colors red, blue, green, yellow, and orange) takes the form

$$
\left[\begin{array}{llllll}
0 & r & b & g & y & o \\
r & 0 & g & y & o & b \\
b & g & 0 & o & r & y \\
g & y & o & 0 & b & r \\
y & o & r & b & 0 & g \\
o & b & y & r & g & 0
\end{array}\right] .
$$

Equating the volumes of all facets with the volume of the first facet leads to the following system of equations (thanks to Maple):

$$
\begin{aligned}
& (g-o)(b-y)(b r-b y-r g+o g+r y-r o)=0, \\
& (y-g)(r-o)(b r-r o+y g-b y+b o-g b)=0, \\
& (y-o)(b-r)(g b-b r+o y-y g+r g-o g)=0, \\
& (r-g)(b-o)(b o-b y+r y-r g+y g-o y)=0, \\
& (r-y)(b-g)(b o-g b+r y-r o+o g-o y)=0 .
\end{aligned}
$$

According to Maple all solutions of this system of equations have the form of three variables set equal while the remaining two are arbitrary.

Thus the partitions with vertex-uniform realizations that are compatible with the equiareal equations are exactly $[3,1,1]$ and its specializations $[3,2],[4,1]$, and [5]. These yield exactly the realizations that are equifacetal! 


\section{The strong center conjecture in dimension 6}

We will, rather laboriously, push the preceding calculations one dimension further. It is presumably possible to carry these calculations into somewhat higher dimensions, but to achieve a general result one will have to find a more abstract and general way of interpreting the equations of equiariality.

7.1 Vertex uniform simplices in dimension 6. First we describe the possible vertexuniform realizations of all the allowable partitions of 6 pointing out which are equifacetal (=vertex-transitive) and which not. In some ways the description is a bit simpler than in dimension 5, because there are only three possible partitions [2, 2, 2], [4, 2], and [6], since all entries in the partitions must be even, as observed in [3]. The partition [6] corresponds to an equilateral simplex.

In either of the other two cases the 2-parts must be realized either by a single 7-cycle or by a 3-cycle together with a disjoint 4-cycle. In the equifacetal case it must be a 7-cycle, by vertex-transitivity.

Up to relabeling there is a unique 7 -cycle. This implies that any realization of $[4,2]$ including a 7-cycle for its 2-part must be a standard one, necessarily equifacetal. Moreover, it follows that there is an essentially unique vertex-uniform realization of $[4,2]$ that is not equifacetal and is characterized by the 2-part consisting of a 3-cycle and a 4-cycle.

It remains to discuss the partition $[2,2,2]$. It is not true that any vertex-uniform realization of $[2,2,2]$ in which all three 2-parts are 7-cycles must be equifacetal. In fact, there is a multiplicity of such edge-colored graphs, which must be enumerated and analyzed. Here is a listing of the resulting cycle structures (CS), up to re-labeling and symmetry:

CS-1: $(1,2,3,4,5,6,7),(1,3,5,7,2,4,6),(1,4,7,3,6,2,5)$

CS-2: $(1,2,3,4,5,6,7),(1,3,5,7,2,6,4),(1,5,2,4,7,3,6)$

CS-3: $(1,2,3,4,5,6,7),(1,3,5,7,4,2,6),(1,4,6,3,7,2,5)$

CS-4: $(1,2,3,4,5,6,7),(1,3,5,2,7,4,6),(1,4,2,6,3,7,5)$

CS-5: $(1,2,3,4,5,6,7),(1,3,6,2,5,7,4),(1,5,3,7,2,4,6)$

CS-6: $(1,2,3,4,5,6,7),(1,3,6,4,2,7,5),(1,4,7,3,5,2,6)$

CS-7: $(1,2,3,4,5,6,7),(1,3,7,4,6,2,5),(1,4,2,7,5,3,6)$

CS-8: $(1,2,3,4,5,6,7),(1,3,7,5,2,4,6),(1,4,7,2,6,3,5)$

Details of the enumeration will only be briefly summarized. The first 7-cycle may be arbitrarily fixed as shown. One creates a list of possible second 7-cycles that contains no successive entries differing by 1 . Perhaps this is best done by focussing on the increments from one entry to the next, the "first differences". Up to re-labeling (action of the dihedral group of order 14) and cyclic permutation it may be assumed that the second 7-cycle in each row starts with 1,3 . (If successive entries in the second 7-cycle all differ by 3 , then the third, remaining, 7-cycle contains entries differing by 2.) For each such pair of 7cycles, one then checks whether the remaining 7 edges form a single cycle. Finally, by hand one eliminates a few duplicates, such as where a particular 7-cycle shows up as the second entry in one row and as the third entry in another row.

We will say that these edge-colorings have coloring type $(7,7,7)$. Only the first one corresponds to an equifacetal 6-simplex or vertex-transitive edge-colored graph, which would have to be invariant under the order 7 cyclic rotation of $(1,2,3,4,5,6,7)$. 
It remains to consider vertex uniform realizations of $[2,2,2]$ in which at least one of the colors gives a 3-cycle and a 4-cycle. (Any such realization gives rise to a realization of $[4,2]$ in which the 2-part consists of a 3-cycle and a 4-cycle, by recoloring the other two parts the same.) We will call such a 2-part consisting of a 3-cycle and a 4-cycle a triangle+square.

There is an essentially unique triangle+square, up to relabeling. If there is a second triangle+square, there is an essentially unique way to add it: the second triangle has one vertex on the first triangle and its other two vertices at diagonally opposite vertices of the first square. Then the quadrilateral that goes with it is essentially unique. And one can check that what is left of $K_{7}$ is a third triangle+square. So we get an essentially unique vertex-uniform realization of $[2,2,2]$ by three triangle+squares. We will say that such a realization has coloring type $(3 / 4,3 / 4,3 / 4)$.

The last possibility is that one has two 7 -cycles and a triangle+square. But starting with a regular 7-gon there is an essentially unique way to add a triangle and then a square (perhaps better described as a "butterfly" or " bowtie"). At that point one can check that what remains is another 7 -gon. So we get an essentially unique vertex-uniform realization of $[2,2,2]$ by two 7 -gons and one triangle+square. We will say that such a realization has coloring type $(7,7,3 / 4)$.

The conclusion is that it suffices to show that the seven non-transitive vertex-uniform realizations of $[2,2,2]$ of coloring type $(7,7,7)$ cannot yield equiareal simplices and that the essentially unique vertex-uniform realizations of $[2,2,2]$ of coloring types $(7,7,3 / 4)$ and $(3 / 4,3 / 4,3 / 4)$ also cannot be equiareal. These calculations will cover the case of the unique vertex-uniform realization of $[4,2]$ that includes a triangle+square, since this is a specialization of the other two. (In the cases of coloring types $(7,7,3 / 4)$ and $(3 / 4,3 / 4,3 / 4)$ the equiareal equations provide additional equations in three unknowns $a, b, c$. Assume that the equiareal equations imply $a=b=c$. Then the equiareal equations where we first set $a=b$ still force $a=b=c$.)

7.2 Equiareal equations in dimension 6. Although we have reduced the study to just one partition type, namely $[2,2,2]$, we have a number of vertex-uniform realizations, and hence a number of distinct versions of the equiareal equations to consider.

We assume that we have a realization with three distinct colors. We will see that, when applied to one of the nonequifacetal vertex-uniform 6-simplices, the equiareal equations almost imply that two of the colors (lengths) must be the same. There is, however, one additional possible "abstract" solution to the equations that one must show cannot come from a simplex. Said another way, there is a solution to the equiareal equations corresponding to a vertex-uniform, edge-colored $K_{7}$ graph, but a separate argument shows that such a solution cannot come from an actual geometric 6-simplex, because of a violation of the triangle inequality.

In the one additional case that might yield a realization of $[4,2]$ with a 2-part consisting of a triangle+square we will see that the equations actually force all colors to be the same.

7.2.1 Coloring type $(7,7,7)$. We only summarize the Maple calculations involved here. In each of the Cases (2) through (8), analysis of the equiareal equations shows that 
either two of the colors must be the same, yielding a known equifacetal coloring with a 7-cycle of one color and everything else of the other color, or the three edge lengths are, up to scaling, $1, \alpha,(\alpha-1)^{2}$ where $\alpha$ is a root of the cubic equation

$$
Z^{3}-5 Z^{2}+6 Z-1=0
$$

This equation has three distinct real solutions. Rounded to five decimal places, the actual triples that occur are $\left(1, \alpha,(\alpha-1)^{2}\right)=$

$$
(1,0.19806,0.643104),(1,1.55496,0.307979) \text {, and }(1,3.24698,5.04892) .
$$

None of the values of $\alpha$ is between 2 and 3, so none can occur by Lemmas 7.1 and 7.2 below.

Lemma 7.1. Any edge-coloring of $K_{7}$ that is a realization of coloring type $(7,7,7)$ contains a triangle with three different edge-colorings.

Proof. Let the three colors be denoted $R, G, B$. There are $\left(\begin{array}{l}7 \\ 3\end{array}\right)$ triangles altogether. There are no triangles with all three edges the same color, since each color forms a 7-cycle. There are 7 triangles with exactly two edges R, 7 with exactly two edges $\mathrm{G}$, and 7 triangles with exactly two edges $\mathrm{B}$, one of each kind at each vertex. This leaves $35-21=14$ triangles with all three colors.

Lemma 7.2. There is a triangle with edge lengths $1, \beta,(\beta-1)^{2}$ if and only if $2<\beta<3$.

Proof. One simply needs to interpret the three triangle inequalities. Further details are omitted.

7.2.2 Coloring type $(\mathbf{7}, \mathbf{7}, \mathbf{3} / \mathbf{4})$. In this case there is an essentially unique vertexuniform coloring realizing it. Analysis of the equiareal equations shows that two of the colors must be the same or one of the lengths degenerates to 0 , contradicting the assumption of three distinct colors or lengths. (More precisely one can observe that the color corresponding to $3 / 4$ must in fact agree with one of the other colors, yielding a known equifacetal coloring of the partition $[4,2]$ with the 2-part realized by an essentially unique 7-cycle.)

7.2.3 Coloring type $(3 / 4,3 / 4,3 / 4)$. This is very similar to the preceding case. Again there is an essentially unique coloring realizing it. But again analysis of the equiareal equations shows that all three colors must then coincide, yielding an equilateral simplex, or one of the lengths must degenerate to 0 .

7.2.4 Comments on partition type $[4,2]$. As already noted, there are up to equivalence two vertex-uniform realizations. One has 2-part a 7-cycle and is vertex-transitive. The other has 2-part a triangle+square and is clearly not vertex-transitive. In the latter case the vertex-uniform realization is a specialization of an edge-coloring of type $(7,7,3 / 4)$ that cannot exist according to the preceding items. 


\section{References}

[1] M. Berger, Geometry. I. Springer 1994. MR1295239 (95g:51001) Zbl 0606.51001

[2] V. Devidé, Über gewisse Klassen von Simplexen. Rad Jugoslav. Akad. Znan. Umjet. 370 (1975), 21-37. MR0477991 (57 \#17487) Zbl 0318.52007

[3] A. L. Edmonds, The geometry of an equifacetal simplex. Mathematika 52 (2005), 31-45 (2006). MR2261840 (2007f:52016) Zbl 1113.52030

[4] A. L. Edmonds, The partition problem for equifacetal simplices. Beiträge Algebra Geom. 50 (2009), 195-213.

[5] A. L. Edmonds, M. Hajja, H. Martini, Coincidences of simplex centers and related facial structures. Beiträge Algebra Geom. 46 (2005), 491-512. MR2196932 (2006k:51020) Zbl 1093.51014

[6] S. G. Krantz, J. E. McCarthy, H. R. Parks, Geometric characterizations of centroids of simplices. J. Math. Anal. Appl. 316 (2006), 87-109. MR2201751 (2006k:52023) Zbl 1089.52009

[7] H. Martini, W. Wenzel, Simplices with congruent $k$-faces. J. Geom. 77 (2003), 136-139. MR2000858 (2004h:52014) Zbl 1031.51012

[8] P. McMullen, Simplices with equiareal faces. Discrete Comput. Geom. 24 (2000), 397-411. MR1758059 (2001f:52025) Zbl 0958.51024

[9] B. L. van der Waerden, Algebra. Vol. I. Springer 1991. MR1080172 (91h:00009a) Zbl 0724.12001

[10] B. Weißbach, Euklidische $d$-Simplexe mit inhaltsgleichen $k$-Seiten. J. Geom. 69 (2000), $227-$ 233. MR1800471 (2001j:52006) Zbl 0965.51009

Received 13 October, 2007; revised 2 June, 2008

A. L. Edmonds, Department of Mathematics, Indiana University, Bloomington, IN 47405, USA

Email: edmonds@indiana.edu 